\title{
MALNUTRITION AND ASSOCIATED MOTOR AND NON-MOTOR FACTORS IN PEOPLE WITH PARKINSON'S DISEASE
}

\author{
Lisette Bazán-Rodríguez ${ }^{1}$, Rossy Cruz-Vicioso ${ }^{2}$, Amin Cervantes-Arriaga ${ }^{3}$, Angel Alcocer-Salas ${ }^{3}$, \\ Daniella Pinto-Solís ${ }^{3}$, ANd MAYela RodríGUeZ-Violante $2,3 *$
}

${ }^{1}$ Neurology Department, ${ }^{2}$ Movement Disorder Clinic, and ${ }^{3}$ Clinical Neurodegenerative Research Unit, Instituto Nacional de Neurología y Neurocirugía Manuel Velazco Suárez, SSA, Mexico City, Mexico

\begin{abstract}
Background: People with Parkinson's disease (PwP) are at higher risk of developing malnutrition. Several factors have been suggested to be involved including motor symptoms, non-motor symptoms, and treatment-related complications. Objective: The objective of the study was to analyze the combined effect of motor, non-motor, and pharmacological factors in the risk of malnutrition in PwP. Methods: Eighty-seven consecutive PwP were included in the study. Clinical data and pharmacological treatment were collected. Nutritional status was assessed using the Mini-Nutritional Assessment (MNA) questionnaire. Movement Disorder Society Unified Parkinson's Disease Rating Scale (MDS-UPDRS), Non-motor Symptoms Scale (NMSS), Hamilton Depression Rating Scale HAM-D, and Montreal Cognitive Assessment were applied. Results: Thirty (34.4\%) PwP were at risk of malnutrition and seven had malnutrition ( $8 \%$ ). Abnormal nutritional status was associated with lower education, higher MDSUPDRS Parts I, II, and III and total scores, and higher scores in the NMSS domain of sleep disorders and fatigue. MDS-UPDRS motor score remained as a determinant of abnormal nutritional status, defined as MNA $<23.5$, with an odds ratio 1.1 (95\% confidence interval 1.01-1.10, $p=0.02$ ). Conclusion: The main factor associated with nutritional status was severity of the motor symptoms as assessed by the MDS-UPDRS Part III. Non-motor symptoms and treatment-related complications were not associated with malnutrition. (REV INVEST CLIN. 2020;72(5):293-9)
\end{abstract}

Key words: Parkinson's disease. Malnutrition. Motor symptoms. Depression. Dementia.

\section{INTRODUCTION}

Parkinson's disease (PD) is one of the most frequent neurodegenerative diseases, with an estimated prevalence of $2 \%$ of adults over age $60^{1}$. People with PD (PwP) present with multiple symptoms including bradykinesia, tremors, and rigidity, but also non-motor symptoms such as anxiety, depression, dysautonomia symptoms, hallucinations, and other problems have been recognized. Some of these symptoms have been reported as important contributing factors to malnutrition in $\mathrm{PwP}^{2}$.
*Corresponding author:

Mayela Rodríguez-Violante

E-mail: mrodriguez@innn.edu.mx
Received for publication: 22-01-2020

Approved for publication: 12-02-2020

DOI: $10.24875 / R I C .20000010$

0034-8376 / (C) 2020 Revista de Investigación Clínica. Published by Permanyer. This is an open access article under the CC BY-NC-ND license (http://creativecommons.org/licenses/by-nc-nd/4.0/). 
Malnutrition is defined as a condition depriving the body of an adequate amount of nutrients. Malnutrition development is a continuum characterized by signs of changes in biochemical indices and body composition $^{3}$.

In the past few years, research in nutritional assessment of PD has exponentially increased. In general, studies have recognized that PWP have a lower body weight and body mass index (BMI) in comparison with healthy controls. The prevalence of malnutrition varies between $0 \%$ and $24 \%$, and the risk of malnutrition between $3 \%$ and $60 \%$ depending on the sample and study design ${ }^{4-6}$.

Several factors related to the disease might contribute to the development of malnutrition. It has been suggested that the main associated factors are the severity of the motor symptoms ${ }^{7}$.

Others have reported that the use of antiparkinsonian medication and their related motor complications such as dyskinesia as well as other drug side effects such as nausea, vomiting, and binge eating might be involved ${ }^{8}$. In addition, other known factors related to lower food intake and weight loss in elderly patients include psychiatric (mainly depression) ${ }^{9}$ and cognitive (mainly dementia) symptoms ${ }^{10}$. Ultimately, malnutrition affects functional ability, quality of life, and sur-

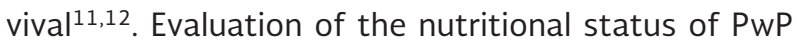
and determination of the malnutrition associated factors is particularly relevant. To the best of our knowledge, no study has assessed all the involved factors as a whole. The aim of the current study is to assess the combined effect of nutritional status with motor, non-motor, and pharmacological factors in PwP.

\section{MATERIALS AND METHODS}

A cross-sectional study was carried out including consecutive PwP attending the National Institute of Neurology and Neurosurgery in Mexico City. PD diagnosis was based on the Movement Disorder Society diagnostic criteria ${ }^{13}$. PwP of any age, medical treatment, or clinical stage were included in the study. Patients who were unable to complete the scales were excluded from the study. Clinical and demographic data regarding gender, age, employment status, current comorbidities (diabetes mellitus, hypertension, and dyslipidemia), weight, height, age at motor onset, and current antiparkinsonian treatment were collected. Anthropometric measurements were calculated such as BMI and levodopa equivalent daily dose (LEDD) was calculated ${ }^{14}$.

The following clinical scales were applied by a neurologist with expertise in movement disorders. The Movement Disorder Society Unified PD Rating Scale (MDS-UPDRS) is comprised four domains: non-motor aspects of daily life (Part I); motor aspects of daily life (Part II); motor exploration (Part III); and motor complications (Part IV) ${ }^{15}$. The non-motor symptom scale (NMSS) assesses nine dimensions: cardiovascular, sleep/fatigue, mood/cognition, perceptual problems/hallucination, attention/memory, gastrointestinal function, urinary function, sexual function, and miscellaneous symptoms ${ }^{16}$. Hoehn and Yahr (HY) stage classifies PwP according to the severity of the disease; Stages 1 and 2 correspond to a mild disease, Stage 3 to moderate disease, and Stages 4 and 5 correspond to a severe disease ${ }^{17}$. The Hamilton Depression Rating Scale (HAM-D) was used for the assessment of depression. A HAM-D score of $<8$ was considered normal and scores $\geq 8$ were considered as depression ${ }^{18}$.

The Montreal Cognitive Assessment (MoCA) assesses cognitive skills with a maximum possible score of 30 points. A cutoff value of $<24$ for cognitive decline has been recommended in Mexican population and was used to classify PwP in two groups (normal cognition and cognitive impairment) $)^{19}$.

Finally, the nutritional assessment was made using the Mini-Nutritional Assessment (MNA). MNA is a validated nutrition screening that can identify patients who are malnourished or at risk of malnutrition, also available and validated in Spanish version ${ }^{20,21}$. The scale includes BMI, weight loss, arm and calf circumference, appetite, antiparkinsonian medication, general and cognitive health, dietary matters, autonomy of feeding, and self-perception of health and nutrition. The maximum score in the questionnaire is 30 . A total score of $<17$ points are considered as "malnutrition"; scores between 17 and 23.5 indicate "at risk for malnutrition"; and scores $\geq 24$ points correspond to "normal nutritional status". For the purposes of this study, patients with scores $<17$ points ("malnutrition") and scores between 17 and 23.5 points ("at risk for 
malnutrition") were merged in one group labeled "abnormal nutritional status."

All subjects gave written informed consent to participate in the study. The study protocol was approved by the local Ethics Committee.

\section{Statistical analysis}

Statistical analysis was performed using the SPSS version 17.0 (IBM SPSS Statistics, SPSS Inc., Chicago, IL). All data are expressed as percentages, means \pm standard deviation, or medians (ranges). KolmogorovSmirnov test was used to analyze the distribution of data, Chi-square test (qualitative data), Mann-Whitney U-test, and Kruskal-Wallis test (quantitative data) were performed as needed. For multivariate analysis, the nutritional status according to the MNA was dichotomized in two groups: malnutrition and non-malnutrition. To identify independent predictors of malnutrition, a multiple logistic regression analysis was performed calculating odds ratio (OR) and $95 \%$ confidence interval $(\mathrm{Cl})$. Independent variables included those that showed statistically significant differences in the bivariate analysis. Multicollinearity was assessed using variation inflation factors (VIFs). Covariables with VIF $>5$ were excluded from the analysis. Hosmer-Lemeshow test was used for goodness of fit. Variance explained by the model was assessed using the Nagelkerke square R. A two-tailed $p<0.05$ was considered as statistically significant.

\section{RESULTS}

A total of 58 males (66.7\%) and 29 females (33.3\%) were included in the study. The mean age was $65.1 \pm$ 12.9 years and the mean disease duration was $7.2 \pm$ 5.5 years. The mean years of schooling were $9.4 \pm$ 5.3 years. Frequency of diabetes mellitus, hypertension, and dyslipidemia was $16.1 \%, 29.9 \%$, and $16.1 \%$, respectively. Regarding anthropometric measurements, the mean weight was $69.6 \pm 13.8 \mathrm{~kg}$, mean height was $160 \pm 18 \mathrm{~cm}$, and the mean $\mathrm{BMl}$ was $27.11 \pm 4.7$. All patients were taking dopaminergic agents at the time of the study, 77 (88.5\%) were on L-dopa, while 47 (54\%) were on dopamine agonists. The mean LEDD was $835.6 \pm 433.4 \mathrm{mg}$.
The total MDS-UPDRS Parts I, II, III, and IV and total were of $10.5 \pm 6.9,12.4 \pm 9.1,34.8 \pm 14.1,3.6 \pm 4.6$, and $53.8 \pm 22.4$ points, respectively. The HAM-D mean score was $6.3 \pm 4.4$, and the mean MoCA score was $24.2 \pm 6.7$. Overall, a total of 31 (35.6\%) subjects were classified with depression and 30 (34.5\%) PwP with cognitive impairment.

Regarding the nutritional score measured by MNA, 49 (56.3\%) patients had normal nutritional status and 37 (42.5\%) had abnormal nutritional status. Of the former, 30 were at risk of malnutrition (34.4\%) and only 7 had malnutrition ( $8 \%$ ). The comparison between the main demographic and clinical characteristics according to the nutritional state is shown in Table 1. Besides the difference in $\mathrm{BMI}$, the only other difference found was in the years of education, with subjects in the malnutrition group having a lower education. No differences were found in the medication use (L-dopa, dopamine agonists) or in LEDD between groups.

Regarding the clinical assessments, PwP with abnormal nutritional status had higher scores in MDS-UPDRS Parts I, II, and III and total scores, as well as in the HY stage. No differences were found in the MoCA and HAM-D scores. The comparison of MDS-UPDRS score, HY stage, MoCA score, and HAM-D score between PwP according to their nutritional state is shown in Table 2.

Regarding the NMSS, higher scores were found in the urinary and mood/cognition domains in the abnormal nutritional status group. On the other hand, perceptual/hallucinations domain had the lowest score. Nevertheless, only the sleep/fatigue domain reached a statistically significant difference between groups with the abnormal nutritional status group scoring higher. The comparison of the NMSS total and individual domain scores according to the nutritional state is shown in Table 2 .

After the logistic regression analysis, only the MDS-UPDRS III remained as a significant predictor of abnormal nutritional state (OR 1.1, 95\% Cl 1.01-1.10, $\mathrm{p}=0.02$ ). The regression model is shown in Table 3.

\section{DISCUSSION}

In this study, the risk of malnutrition was detected in $35.6 \%$ of the patients and malnutrition in $8 \%$. 
Table 1. Comparison of the main demographic and clinical characteristics in patients with Parkinson's disease according to their nutritional state

\begin{tabular}{lccc}
\hline Variable & $\begin{array}{c}\text { Normal nutritional state } \\
(\mathbf{n}=49)\end{array}$ & $\begin{array}{c}\text { Abnormal nutritional state } \\
(\mathbf{n}=38)\end{array}$ & $\mathrm{p}$ \\
\hline Age & $64.4 \pm 12.3$ & $66 \pm 13.9$ & 0.58 \\
Male gender & $35(71.4 \%)$ & $23(60.5 \%)$ & 0.29 \\
Years of education & $10.4 \pm 5.8$ & $8.1 \pm 4.3$ & 0.04 \\
Body mass index & $28.1 \pm 4.9$ & $25.8 \pm 4.3$ & 0.02 \\
Disease duration & $7.6 \pm 5.6$ & $6.7 \pm 5.6$ & 0.35 \\
Use of L-dopa & $44(89.7 \%)$ & $36(94.7 \%)$ & 0.41 \\
Use of dopamine agonist & $27(55 \%)$ & $20(52.6 \%)$ & 0.82 \\
LEDD & $815.3 \pm 485.1$ & $834.9 \pm 448.4$ & 0.45 \\
Diabetes mellitus & $10(20.4 \%)$ & $4(10.5 \%)$ & 0.21 \\
Hypertension & $16(32.7 \%)$ & $10(26.3 \%)$ & 0.52 \\
Dyslipidemia & $11(22.4 \%)$ & $3(7.9 \%)$ & 0.07 \\
Depression & $15(30.6 \%)$ & $15(39.5 \%)$ & 0.51 \\
Cognitive impairment & $14(28.6 \%)$ & $16(42.1 \%)$ & 0.18 \\
\hline
\end{tabular}

HAM-D score > 8; MoCA score <24; LEDD, levodopa equivalent daily dose; MoCA, Montreal Cognitive Assessment.

Fereshtehnejad et al., in their case-control study, reported a combined prevalence of malnutrition and risk of malnutrition of $40 \%$, with a sample of patients similar to ours ${ }^{22}$. Our study is consistent with the review literature reported in the previous studies based on MNA assessment, which shows the prevalence of up to $20-45 \%$ of malnutrition ${ }^{23}$.

We examined all known potential contributing factors with the context of PD, including motor and nonmotor symptoms, depression, cognitive decline, and use of antiparkinsonian medications like dopamine agonists. All these factors have been associated with nutritional status in PwP24.

As expected, anthropometrics measurements showed significant differences between PwP with abnormal nutrition status in comparison to those with normal status. BMI, weight, and height were lower for the former group. Demographic variables such as age, gender, disease duration, and comorbidities were not related to nutritional status in our sample. Interestingly, PwP and abnormal nutritional status had lower years of education. Previously, an Italian study reported that poverty is strongly associated with lower levels of education, therefore, leading to greater difficulties in achieving minimum nutritional needs ${ }^{25}$.
On the other hand, motor and non-motor symptoms were significantly associated with nutritional status in our sample of PwP. PwP with abnormal nutritional status had more severe motor symptoms as assessed by the selected scales. Furthermore, disease severity was worst as assessed by the HY; this association has been previously reported ${ }^{12,24}$. MDS-UPDRS scores were also higher in the abnormal nutritional status group in all parts except for Part IV. This association between UPDRS (a previous version of the MDS-UPDRS) scores has also been previously reported ${ }^{26}$. Nevertheless, association with Part IV is usually reported in the literature. Both dyskinesia and wearing-off phenomenon have been associated with the risk of malnutrition. In fact, the existence of a Park-weight phenotype has been suggested, in which patients with higher levodopa dose are at a higher risk of dyskinesia and weight $\operatorname{loss}^{27}$. Interestingly, our study sample did not differ significantly in terms of LEDD and weight in comparison to other reports, but the frequency of dyskinesia was lower, whereas this difference is inherent to Mexican population warrants further study.

Regarding non-motor symptoms, gastrointestinal dysfunction may lead to malnutrition due to insufficient intake or impaired absorption, along with the increased energy expenditure associated with the 
Table 2. Comparison of the main clinical scales between persons with Parkinson's disease according to their nutritional state

\begin{tabular}{|c|c|c|c|}
\hline Variable & $\begin{array}{l}\text { Normal nutritional state } \\
\qquad(n=49)\end{array}$ & $\begin{array}{l}\text { Abnormal nutritional state } \\
\qquad(\mathrm{n}=38)\end{array}$ & $\mathrm{p}$ \\
\hline MDS-UPDRS I & $8.8 \pm 6$ & $12.8 \pm 7.4$ & 0.003 \\
\hline MDS-UPDRS II & $10 \pm 7.5$ & $15.6 \pm 10$ & 0.009 \\
\hline MDS-UPDRS III & $30.4 \pm 11.5$ & $40.5 \pm 15.2$ & 0.001 \\
\hline MDS-UPDRS IV & $3.5 \pm 4.6$ & $3.7 \pm 4.7$ & 0.71 \\
\hline MDS-UPDRS total & $46.2 \pm 17.9$ & $63.6 \pm 24$ & 0.001 \\
\hline Hoehn and Yahr & $2.1 \pm 0.5$ & $2.4 \pm 0.6$ & 0.04 \\
\hline NMSS cardiovascular & $1 \pm 1.9$ & $2 \pm 3.0$ & 0.11 \\
\hline NMSS sleep/fatigue & $5.4 \pm 7.8$ & $13.2 \pm 12.5$ & 0.002 \\
\hline NMSS mood/cognition & $9.3 \pm 7.1$ & $9.9 \pm 9.9$ & 0.76 \\
\hline $\begin{array}{l}\text { NMSS perceptual } \\
\text { problems/hallucinations }\end{array}$ & $0.7 \pm 1.8$ & $0.7 \pm 2.0$ & 0.60 \\
\hline NMSS attention/memory & $3.8 \pm 5.1$ & $5.9 \pm 8.0$ & 0.15 \\
\hline NMSS gastrointestinal & $5.1 \pm 5.2$ & $7.3 \pm 7.6$ & 0.24 \\
\hline NMSS urinary & $11 \pm 9.8$ & $13.2 \pm 11.3$ & 0.43 \\
\hline NMSS sexual function & $1.9 \pm 3.4$ & $3.1 \pm 6.6$ & 0.76 \\
\hline NMSS miscellaneous & $7.3 \pm 6.6$ & $8.6 \pm 6.4$ & 0.23 \\
\hline NMSS total & $48.07 \pm 29.55$ & $60.51 \pm 37.60$ & 0.16 \\
\hline MNA & $26.4 \pm 1.5$ & $19.8 \pm 3.2$ & $<0.001$ \\
\hline MoCA & $25.2 \pm 6.2$ & $22.9 \pm 7.2$ & 0.17 \\
\hline HAM-D & $5.8 \pm 3.9$ & $7 \pm 4.9$ & 0.29 \\
\hline
\end{tabular}

MDS-UPDRS: Movement Disorders Society Unified Parkinson's Disease Rating Scale; MNA: Mini-Nutritional Assessment; NMSS: Non-Motor Symptoms Scale; HAM-D: Hamilton Depression; MoCA: Montreal Cognitive Assessment; NMSS: Non-Motor Symptoms Scale.

Table 3. Logistic regression analysis to identify independent predictors of malnutrition

\begin{tabular}{lcccc}
\hline Variable & B & Odds ratio & $95 \% \mathrm{Cl}$ & $\mathrm{p}$ \\
\hline Years of education & -0.49 & 0.95 & $0.86-1.05$ & 0.33 \\
MDS-UPDRS I & 0.09 & 1.09 & $0.99-1.19$ & 0.07 \\
MDS-UPDRS II & 0.02 & 1.02 & $0.95-1.10$ & 0.55 \\
MDS-UPDRS III & 0.50 & 1.10 & $1.01-1.10$ & 0.02 \\
NMSS Sleep/fatigue & -0.36 & 0.97 & $0.90-1.04$ & 0.35 \\
\hline
\end{tabular}

MDS-UPDRS: Movement Disorders Society Unified Parkinson's Disease Rating Scale; NMSS: Non-Motor Symptoms Scale. Cl: confidence interval.

movement disorder. In our study, we did not find an association with gastrointestinal symptoms such as difficulty swallowing and constipation. This can be partially explained by the fact that symptoms such as slow gastric emptying, early satiety, and postprandial fullness are not assessed by the NMSS. Unexpectedly, we found a higher prevalence of sleep/fatigue and urinary symptoms in the PwP with abnormal nutritional status. Interestingly, an association between serum concentrations of micronutrients and malnutrition with lower urinary tract symptoms has been previously described in the elderly ${ }^{28}$. On the other hand, some epidemiologic studies reported associations between dietary patterns and sleep quality investigating the effect of concentrations of macronutrients on sleep architecture and fatigue ${ }^{29}$. To the 
best of our knowledge, these associations have not been previously described in PwP.

The type and dose of antiparkinsonian medication as part of the risk of malnutrition as reported in other studies such as Laudisio et al. ${ }^{8}$ they reported that total LEDD was associated with a worse nutritional status. In addition, the use of D2 dopamine agonists, such as pramipexole, has been associated with body weight increase. ${ }^{30}$ It has also been hypothesized that the need of several daily administrations in fasting conditions could favor malnutrition as well as the direct effect of L-dopa on fat metabolism ${ }^{31}$. Conversely, other studies in Asian population found no difference in the use of dopaminergic agents and the presence of dyskinesia or in the association with nutritional status ${ }^{6,12}$. In the present study, the use of dopaminergic agents was not related to nutritional status, in line with these results. Furthermore, although L-dopa use and total LEDD were higher in our PwP with malnutrition population, no statistically significant association was found.

Due to its chronic nature, PD often exerts severe psychological effects, leading to insufficient nutrient intake and exposing the patients to the risk of malnutrition. Fereshtehnejad et al. highlighted the relevance of nutritional status as a contributor to quality of life and general conditions of daily living in PwP26. Their results indicate that, in addition to motor symptoms, emotional well-being and depression were associated with an abnormal nutritional status. In our study, the prevalence of depression as assessed by the HAM-D was not different between groups. It has been reported that nutritional status is worse in subjects with dementia both in tauopathies (frontotemporal lobar degeneration) and alpha-synucleinopathies (dementia with Lewy bodies) ${ }^{32}$. In addition, we did not find any statistically significant difference in cognitive function between groups. After multivariate regression analysis, the only variable that remained in the model with a statistically significant difference was the MDS-UPDRS Part III.

The present study has several limitations. The interpretation and applicability of the results should be considered thoroughly as well as the, limited sample size and cross-sectional design. Cognitive decline was assessed using the MoCA, which is a validated screening tool but does not substitute a comprehensive neuropsychological assessment. Dysphagia is assessed in the MDS-UPDRS Part II, but the use of a specific scale for this purpose would be better suited. It should also be mentioned that while the frequency of some comorbidities was collected, their actual control was not assessed. Moreover, data on some other chronic conditions such as liver or kidney disease were not collected. External validation should also be interpreted cautiously since the study was carried at a tertiary hospital, thus a referral bias cannot be ruled out.

The only factor associated with abnormal nutritional status was the severity of the motor symptoms as assessed by the MDS-UPDRS Part III and the disease severity according to the HY stage. Non-motor symptoms, including depression and cognitive decline, as well as antiparkinsonian drugs and dose were not related to an abnormal nutritional state defined as risk of malnutrition or malnutrition.

\section{REFERENCES}

1. Jankovic J. Parkinson's disease: clinical features and diagnosis. J Neurol Neurosurg Psychiatry. 2008;79:368-76.

2. Sheard JM, Ash S, Silburn PA, Kerr GK. Prevalence of malnutrition in Parkinson's disease: a systematic review. Nutr Rev. 2011; 69:520-32

3. Vellas B, Guigoz Y, Garry PJ, Nourhashemi F, Bennahum D, Lauque $S$, et al. The mini nutritional assessment (MNA) and its use in grading the nutritional state of elderly patients. Nutrition. 1999;15:116-22

4. Barichella M, Villa MC, Massarotto A, Cordara SE, Marczewska A, Vairo A, et al. Mini nutritional assessment in patients with Parkinson's disease: correlation between worsening of the malnutrition and increasing number of disease-years. Nutr Neurosci. 2008;11:128-34.

5. Barichella M, Cereda E, Pezzoli G. Major nutritional issues in the management of Parkinson's disease. Mov Disord. 2009;24: 1881-92.

6. Kim SR, Chung SJ, Yoo SH. Factors contributing to malnutrition in patients with Parkinson's disease. Int J Nurs Pract. 2016; 22:129-37.

7. Uc EY, Struck LK, Rodnitzky RL, Zimmerman B, Dobson J, Evans WJ. Predictors of weight loss in Parkinson's disease. Mov Disord. 2006;21:930-6.

8. Laudisio A, Vetrano DL, Meloni E, Ricciardi D, Franceschi F, Bentivoglio AR, et al. Dopaminergic agents and nutritional status in Parkinson's disease. Mov Disord. 2014;29:1543-7.

9. Pérez Cruz E, Lizárraga Sánchez DC, Martínez Esteves Mdel R. [Association between malnutrition and depression in elderly]. Nutr Hosp. 2014;29:901-6

10. Sanders CL, Wengreen HJ, Schwartz S, Behrens SJ, Corcoran C, Lyketsos CG, et al. Nutritional status is associated with severe dementia and mortality: the cache county dementia progression study. Alzheimer Dis Assoc Disord. 2018;32:298-304.

11. Ongun N. Does nutritional status affect Parkinson's disease features and quality of life? PLoS One. 2018;13:e0205100.

12. Wang G, Wan Y, Cheng Q, Xiao Q, Wang Y, Zhang J, et al. Malnutrition and associated factors in Chinese patients with Parkinson's disease: results from a pilot investigation. Parkinsonism Relat Disord. 2010;16:119-23.

13. Tomlinson CL, Stowe R, Patel S, Rick C, Gray R, Clarke CE. Systematic review of levodopa dose equivalency reporting in Parkinson's disease. Mov Disord. 2010;25:2649-53. 
14. Postuma RB, Berg D, Stern M, Poewe W, Olanow CW, Oertel W, et al. MDS clinical diagnostic criteria for Parkinson's disease. Mov Disord. 2015;30:1591-601.

15. Martinez-Martin P, Rodriguez-Blazquez C, Alvarez-Sanchez M, Arakaki T, Bergareche-Yarza A, Chade A, et al. Expanded and independent validation of the movement disorder society-unified Parkinson's disease rating scale (MDS-UPDRS). J Neurol. 2013;260:228-36

16. Chaudhuri KR, Martinez-Martin P, Brown RG, Sethi K, Stocchi F, Odin $\mathrm{P}$, et al. The metric properties of a novel non-motor symptoms scale for Parkinson's disease: results from an international pilot study. Mov Disord. 2007;22:1901-11.

17. Martinez-Martin P, Skorvanek M, Rojo-Abuin JM, Gregova Z, Stebbins GT, Goetz CG, et al. Validation study of the hoehn and yahr scale included in the MDS-UPDRS. Mov Disord. 2018;33:651-2.

18. Hamilton M. A rating scale for depression. J Neurol Neurosurg. Psychiatry. 1960;23:56-62.

19. Aguilar-Navarro SG, Mimenza-Alvarado AJ, Palacios-García AA, Samudio-Cruz A, Gutiérrez-Gutiérrez LA, Ávila-Funes JA. Validity and reliability of the Spanish version of the Montreal cognitive assessment (MoCA) for the detection of cognitive impairment in Mexico. Rev Colomb Psiquiatr. 2018;47:237-43.

20. Kaiser MJ, Bauer JM, Ramsch C, Uter W, Guigoz Y, Cederholm $\mathrm{T}$, et al. Validation of the mini nutritional assessment short-form (MNA-SF): a practical tool for identification of nutritional status. J Nutr Health Aging. 2009;13:782-8

21. Ghazi L, Fereshtehnejad SM, Abbasi Fard S, Sadeghi M, Shahidi GA, Lökk J. Mini nutritional assessment (MNA) is rather a reliable and valid instrument to assess nutritional status in Iranian healthy adults and elderly with a chronic disease. Ecol Food Nutr. 2015;54:342-57.

22. Fereshtehnejad SM, Ghazi L, Sadeghi M, Khaefpanah D, Shahidi GA, Delbari A, et al. Prevalence of malnutrition in patients with Parkinson's disease: a comparative study with healthy controls using mini nutritional assessment (MNA) questionnaire. J Parkinsons Dis. 2014;4:473-81.
23. Tomic S, Pekic V, Popijac Z, Pucic T, Petek M, Kuric TG et al. What increases the risk of malnutrition in Parkinson's disease? J Neurol Sci. 2017;375:235-8

24. Jaafar AF, Gray WK, Porter B, Turnbull EJ, Walker RW. A crosssectional study of the nutritional status of community-dwelling people with idiopathic Parkinson's disease. BMC Neurol. 2010; 10:124

25. Donini LM, Scardella P, Piombo L, Neri B, Asprino R, Proietti AR, et al. Malnutrition in elderly: social and economic determinants. J Nutr Health Aging. 2013;17:9-15.

26. Fereshtehnejad SM, Ghazi L, Shafieesabet M, Shahidi GA, Delbari A, Lökk J. Motor, psychiatric and fatigue features associated with nutritional status and its effects on quality of life in Parkinson's disease patients. PLoS One. 2014;9:e91153.

27. Sharma JC, Vassallo M. Prognostic significance of weight changes in Parkinson's disease: the park-weight phenotype. Neurodegener Dis Manag. 2014;4:309-16.

28. Rohrmann S, Smit E, Giovannucci E, Platz EA, Third National Health and Nutrition Examination Survey. Association between serum concentrations of micronutrients and lower urinary tract symptoms in older men in the Third National Health and Nutrition Examination Survey. Urology. 2004;64:504-9.

29. St-Onge MP, Mikic A, Pietrolungo CE. Effects of diet on sleep quality. Adv Nutr. 2016;7:938-49

30. Kumru H, Santamaria J, Valldeoriola F, Marti MJ, Tolosa E. Increase in body weight after pramipexole treatment in Parkinson's disease. Mov Disord. 2006;21:1972-4.

31. Cooper S], Al-Naser HA, Clifton PG. The anorectic effect of the selective dopamine D1-receptor agonist A-77636 determined by meal pattern analysis in free-feeding rats. Eur J Pharmacol. 2006;532:253-7.

32. Koyama A, Hashimoto $M$, Tanaka $H$, Fujise $N$, Matsushita $M$ Miyagawa $Y$, et al. Malnutrition in Alzheimer's disease, dementia with Lewy bodies, and frontotemporal lobar degeneration: comparison using serum albumin, total protein, and hemoglobin level. PLoS One. 2016;11:e0157053. 